\title{
UNC-45A Is Highly Expressed in the Proliferative Cells of the Mouse Genital Tract and in the Microtubule-Rich Areas of the Mouse Nervous System
}

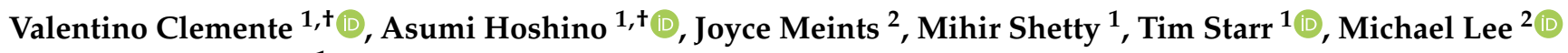 \\ and Martina Bazzaro ${ }^{1, *}$ \\ 1 Masonic Cancer Center and Department of Obstetrics, Gynecology and Women's Health, \\ University of Minnesota, Minneapolis, MN 55455, USA; vclement@umn.edu (V.C.); \\ hoshi020@umn.edu (A.H.); shett036@umn.edu (M.S.); star0044@umn.edu (T.S.) \\ 2 Department of Neuroscience, University of Minnesota, Minneapolis, MN 55455, USA; \\ meint002@umn.edu (J.M.); mklee@umn.edu (M.L.) \\ * Correspondence: mbazzaro@umn.edu; Tel.: +1-612-625-2889; Fax: +1-612-626-0665 \\ + Equal contributors.
}

Citation: Clemente, V.; Hoshino, A.; Meints, J.; Shetty, M.; Starr, T.; Lee, M.; Bazzaro, M. UNC-45A Is Highly

Expressed in the Proliferative Cells of the Mouse Genital Tract and in the Microtubule-Rich Areas of the Mouse Nervous System. Cells 2021, 10, 1604. https://doi.org/10.3390/cells10071604

Academic Editor: Alfredo Gorio

Received: 20 May 2021

Accepted: 21 June 2021

Published: 26 June 2021

Publisher's Note: MDPI stays neutral with regard to jurisdictional claims in published maps and institutional affiliations.

Copyright: (C) 2021 by the authors Licensee MDPI, Basel, Switzerland. This article is an open access article distributed under the terms and conditions of the Creative Commons Attribution (CC BY) license (https:/ / creativecommons.org/licenses/by/ $4.0 /)$.

\begin{abstract}
UNC-45A (Protein unc-45 homolog A) is a cytoskeletal-associated protein with a dual and non-mutually exclusive role as a regulator of the actomyosin system and a Microtubule (MT)destabilizing protein, which is overexpressed in human cancers including in ovarian cancer patients resistant to the MT-stabilizing drug paclitaxel. Mapping of UNC-45A in the mouse upper genital tract and central nervous system reveals its enrichment not only in highly proliferating and prone to remodeling cells, but also in microtubule-rich areas, of the ovaries and the nervous system, respectively. In both apparatuses, UNC-45A is also abundantly expressed in the ciliated epithelium. As regulators of actomyosin contractility and MT stability are essential for the physiopathology of the female reproductive tract and of neuronal development, our findings suggest that UNC-45A may have a role in ovarian cancer initiation and development as well as in neurodegeneration.
\end{abstract}

Keywords: UNC-45A; ovaries; neurons; brain; cilia; microtubules

\section{Introduction}

UNC-45A (Protein unc-45 homolog A) is a member of the UCS (UNC-45/CRO1/She4p) protein family [1,2], with dual and non-mutually exclusive roles as a regulator of the actomyosin system [3,4] and a microtubule (MT)-destabilizing protein [5-7]. Regulators of actomyosin contractility and MT stability are essential for both ovarian cancer cell proliferation and neuronal development. For instance, dysregulation of the Rho/ROCK (Rho-associated protein kinase) signaling pathway is commonly found in ovarian cancer [8-11] and implicated in the pathophysiology of the nervous system [12,13]. Several MT-destabilizing proteins are also expressed in both neurons [14-18] and cancer cells, including ovarian cancer cells [19-21], where they play roles spanning from regulating symmetrical and asymmetrical cell division [22] to regulating MT mass [6] and sensitivity to MT-targeting agents $[6,23]$. As a key regulator of cytoskeletal activities, UNC-45A participates in several cellular functions including cytokinesis [24-27], exocytosis [28], and axonal growth [29]. UNC-45A is overexpressed in breast and ovarian cancer as compared to their normal counterpart [24-26] and in ovarian cancer patients that are resistant to the MT-stabilizing drug paclitaxel [6].

In light of the role played by UNC-45A in regulating cell proliferation and of its role as a MAP with MT-destabilizing properties, the objective of this study was to determine the pattern of UNC-45A expression in cells of the mouse genital tract with different proliferative and remodeling capacities and in the mouse central nervous system (CNS). 
We found that UNC-45A is enriched in the highly proliferating and prone to remodeling cells of the mouse reproductive system, in the microtubule-rich regions of the mouse central nervous system and in the cilia of both, the mouse reproductive system and the brain. Taken together, these findings suggest that UNC-45A may play a role in the physiology and pathology of the female reproductive apparatus and the central nervous system.

\section{Materials and Methods}

\subsection{Sample Preparation of Mouse CNS and Upper Genital Tract}

Ovaries, fallopian tubes, brains, and spinal cords from adult C57BL/6 mice were preserved in $10 \%$ formalin buffer solution for $24 \mathrm{~h}$ followed by $70 \%$ ethanol to prevent the tissue from decomposing, and embedded in paraffin. At least two different mice were used to evaluate the UNC-45A expression level in CNS and mouse upper genital tract. The phase of the estrous cycle was determined by histological evaluation as previously described [30,31].

\subsection{Antibodies}

Anti-UNC-45A Rabbit Polyclonal antibody (19564-1-AP, Protein Tech, Rosemont, IL, USA) was used for IHC and IF. The specificity of this antibody has been previously validated by us and others by Western Blot, IF, and IHC analyses [5,6]. The Anti-NeuN mouse monoclonal antibody (MA5-33103, ThermoFisher, Waltham, MA, USA) was used for IF. Secondary antibodies for IF analyses were FITC-conjugated Goat Anti-Rabbit IgG used at 1:200 dilution (Jackson ImmunoResearch Laboratories, West Grove, PA, USA) and TexasRed conjugated Goat Anti-Mouse IgG used at 1:200 dilution (Jackson ImmunoResearch Laboratories, West Grove, PA, USA).

\subsection{Immunohistochemical Staining of Mouse Upper Genital Tract, Brain, and Spinal Cord}

Five $\mu \mathrm{m}$ formalin-fixed; paraffin-embedded (FFPE) tissue sections were subjected to hematoxylin and eosin staining and immunohistochemistry (IHC) for UNC-45A. Slides were deparaffinized with $100 \%$ xylene and rehydrated with gradient ethanol $(100 \%, 95 \%$, and $80 \%$ ). Antigen retrieval was then carried out with 1X Reveal Decloaker (Biocare Medical, Pacheco, CA, USA) in a vegetable steamer for $30 \mathrm{~min}$ at $100{ }^{\circ} \mathrm{C}$, before blocking the slides with Background Sniper (BS966H, Biocare Medical, Pacheco, CA, USA) for $13 \mathrm{~min}$ at room temperature. Sections were subsequently incubated with anti-UNC45A antibody at dilution of 1:100 for mouse brain and spinal cord and 1:200 for mouse upper genital tract overnight at $4{ }^{\circ} \mathrm{C}$, followed by Biotin-SP-conjugated AffiniPure Goat AntiRabbit IgG (111-065-003, Jackson ImmunoResearch Laboratories, West Grove, PA, USA) at a dilution of 1:200 for $30 \mathrm{~min}$ at room temperature and incubation with horseradish peroxidase streptavidin at a dilution of 1:125 (405210, BioLegend, San Diego, CA, USA) for $30 \mathrm{~min}$ at room temperature. After the staining was developed with 3,3'-diaminobenzidine (926506, BioLegend, San Diego, CA, USA) for $3 \mathrm{~min}$, the slides were counterstained with Harris' hematoxylin.

\subsection{Bright-Field Imaging}

Brightfield images were acquired with a Zeiss Axio Scan.Z1 system (Zeiss, Thornwood, NY, USA) at $40 \times$ magnification. Images of details were taken with a BX40 light microscope, DP72 camera, and cellSens Standard v1.16 imaging software (Olympus, Waltham, MA, USA).

\subsection{Immunofluorescence (IF) Microscopy}

For immunofluorescence analysis of UNC-45A and NeuN (neuronal nuclear protein) in a mouse brain, $5 \mu \mathrm{m}$ formalin-fixed; paraffin embedded (FFPE) tissue sections were deparaffinized, rehydrated, retrieved, and blocked similarly to what described in the immunohistochemical staining section. Sections were then incubated with anti-UNC45A and anti-NeuN at the indicated concentration overnight at $4{ }^{\circ} \mathrm{C}$. Secondary antibodies were 
used at the indicated concentration for $1 \mathrm{~h}$ at room temperature and sections were mounted with mounting media containing DAPI (4',6-diamidino-2-phenylindole) (F6057 SigmaAldrich. Burlington, MA, USA). Images were obtained on an Axiovert 200 microscope (Zeiss, Thornwood, NY, USA) equipped with a high-resolution CCD camera.

\subsection{Quantification of UNC-45A Expression Levels via Immunofluorescence and via Immunohistochemistry}

Quantification of immunohistochemistry was done on ImageJ using a slightly modified version of a previously described protocol [32]. Briefly, using whole slide scansions, regions of interest (ROIs) were drawn around the borders of the single elements analyzed before saving them into the ROI manager. Images were then converted into their RGB format and colors deconvolved using the color deconvolution function of ImageJ. Finally, the DAB signal was thresholded with a fixed upper limit of 70 , and the mean grey values representing pixel intensity were obtained from individual ROIs. For quantification of immunofluorescence, pixel intensity was measured from the green channel after drawing the ROIs, and the background was subtracted without further image processing. At least three different areas were measured for each histological element and data compared using GraphPad Prism (version 8.4.3, San Diego, CA, USA).

\section{Results}

3.1. UNC-45A Is Expressed in the Mouse Upper Genital Tract with a Stronger Expression in Proliferating Cells and Cilia

We and others have previously shown that UNC-45A is expressed in human ovarian surface epithelial cells and human breast cells and overexpressed in highly proliferative cells of ovarian and breast cancers as compared to their normal counterpart [24,25]. Here, we performed H\&E (hematoxylin and eosin) and UNC-45A staining (Figure 1A, upper and lower panels, respectively) in mouse ovaries and fallopian tubes (diestrus) to determine UNC-45A expression pattern in the mouse upper genital tract during diestrus. We found that UNC-45A is abundantly expressed in cells of the early follicles (Figure 1B, i), the corpus luteum (Figure 1B, ii), and the later follicles (Figure 1B, iii).

Furthermore, UNC-45A is strongly expressed in the oocytes (Figure 1B black asterisks) and the ovarian surface epithelium (Figure 1B, inset, black arrow), while the stroma is mostly negative. In the fallopian tubes, UNC-45A is weakly expressed in the smooth muscle cells and more intensively expressed in the fallopian tube epithelium and in particular in the cilia of the fallopian tube epithelium (Figure 1C and its inset, green arrows). Quantification of UNC-45A levels in the ovary and fallopian tube during diestrus as determined by immunohistochemistry are given in Figure 2 (left). Importantly, cilia are microtubule (MT)based structures capable of moving themselves using dynein contractility, and we have recently shown that UNC-45A is a Microtubule-Associated-Protein (MAP) responsible for modulating MT dynamics $[7,33,34]$. Taken together, this suggests that in the mouse upper genital tract UNC-45A expression is stronger in highly proliferative and prone to remodeling cells and within that UNC-45A may play a role in regulating cilia functions.

We also performed H\&E and UNC-45A staining of the mouse ovary and fallopian tube during proestrus. This phase was chosen because, while the follicles of short cycling animals like mice are constantly proliferating, the element of the mouse ovary mostly affected by significant changes in proliferation and activity during different phases is represented by the corpus luteum $[30,31]$, which degenerates during proestrus. We found no drastic changes in the UNC-45A expression pattern, with all the previous structures being strongly stained by UNC-45A (Figure 1D and its insets). Of note, as compared to the diestrus, more mature follicles displayed a slightly more intense staining than their earlier counterparts (Figure 1E, i and iii). Corpora lutea, which are degenerating prevalently in this phase, displayed slightly decreased expression of UNC-45A (Figure 1E, ii). No changes were observed in the surface epithelium (Figure 1E and its inset, black arrow), the fallopian tube (Figure $1 \mathrm{~F}$ and its inset) and the cilia (Figure $1 \mathrm{~F}$ and its inset, green arrows). 
Quantification of UNC-45A levels in the ovary and fallopian tube during proestrus as determined by immunohistochemistry are given in Figure 2 (right).

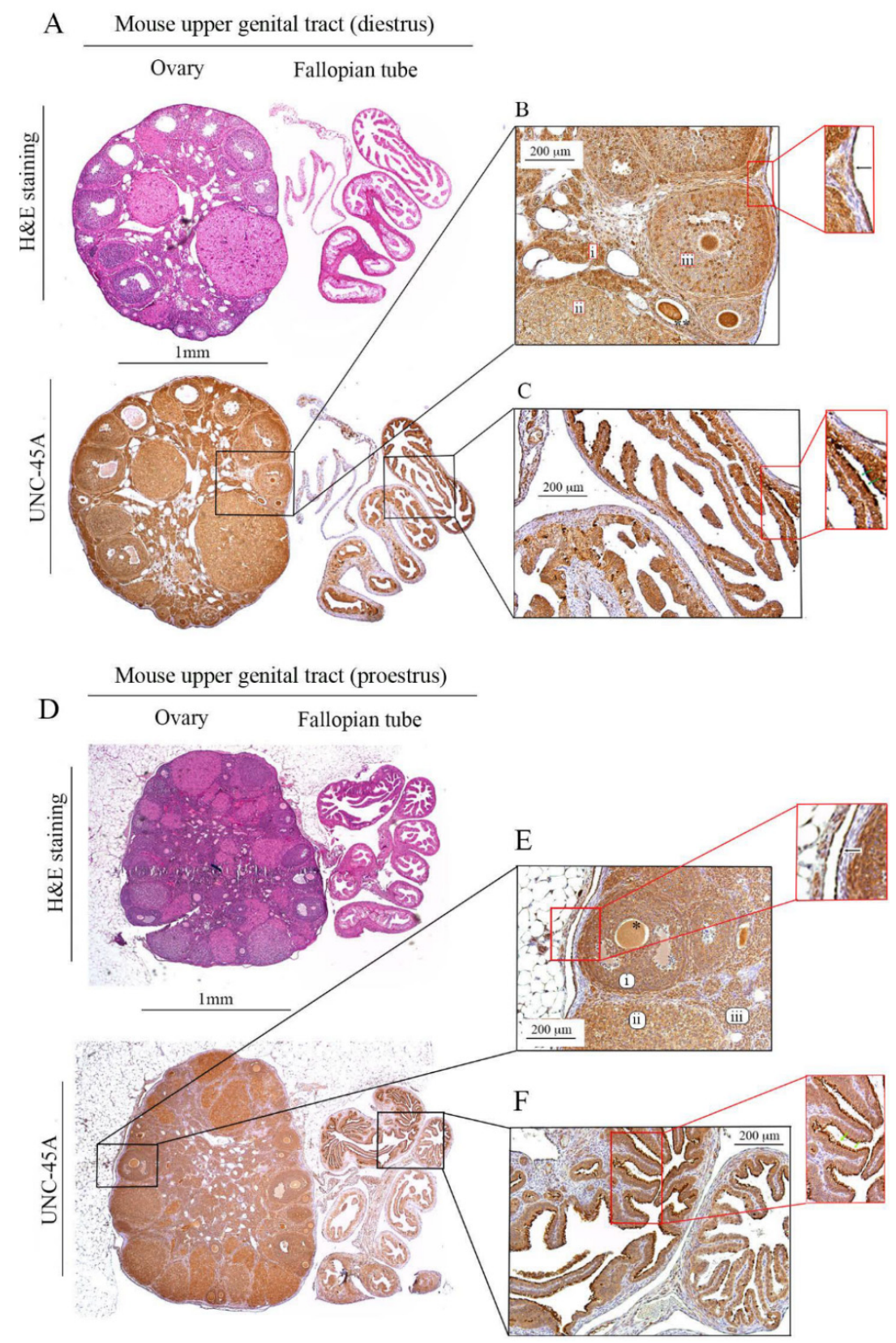

Figure 1. UNC-45A (Protein unc-45 homolog A) expression in mouse ovaries and fallopian tube during diestrus and proestrus. (A). H\&E (hematoxylin and eosin) (upper panel) and UNC-45A (lower panel) staining of the mouse ovary and fallopian tube during diestrus (B). Details of mouse ovary with follicular structures strongly stained for UNC-45A (i. early follicles, ii. corpus luteum, and iii. later follicles) and containing oocytes (asterisks). Inset, mouse ovarian surface epithelium (arrow) positive for UNC-45A while the stroma is negative. (C). Details of mouse fallopian tube with epithelium strongly stained for UNC-45A. Inset, cilia in fallopian tube epithelium (green arrows) strongly positive for UNC-45A. (D). H\&E (upper panel) and UNC-45A (lower panel) staining of the mouse ovary and fallopian tube during proestrus (E). Details of mouse ovary with follicular structures strongly stained for UNC-45A (i. later follicles, ii. corpus luteum, and iii. early follicles) and containing oocytes (asterisks). Inset, mouse ovarian surface epithelium (arrow) positive for UNC-45A while the stroma is negative. (F). Details of mouse fallopian tube with epithelium strongly stained for UNC-45A. Inset, cilia in fallopian tube epithelium (green arrows) strongly positive for UNC-45A. 

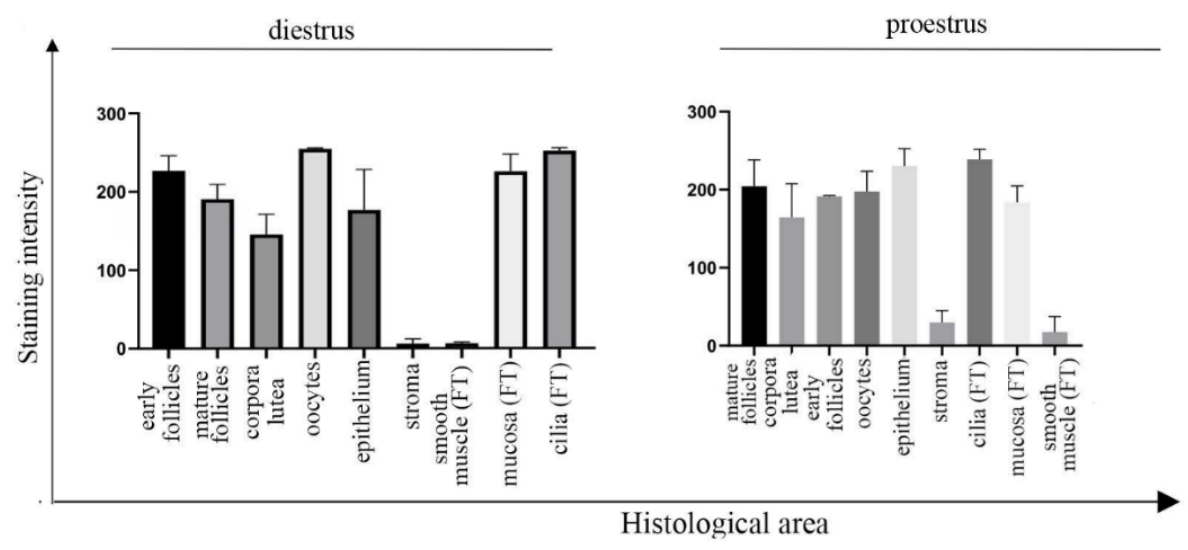

Figure 2. Quantification of UNC-45A expression levels in mouse ovaries and fallopian tubes (FTs) during diestrus and proestrus following IHC staining. Quantification of UNC-45A staining intensity in the single components of the ovary and fallopian tube. Quantification is expressed as mean pixel intensity, during diestrus (left) and proestrus (right).

\subsection{UNC-45A Is Expressed in the Microtubule-Rich Regions of the Mouse Central Nervous System (CNS) and Mouse Nerve Roots}

We have recently shown that UNC-45A is a Microtubule-Associated-Protein (MAP) with MT-severing properties [5-7], which is important for neurite development [29]. In neurons, MTs play a pivotal role in both the structure and the function of axons and dendrites, whereas MAPs have been extensively shown to be major regulators of the physiology and pathology of the nervous system $[35,36]$. Here, we performed H\&E and UNC-45A staining (Figure 3A,B, respectively) of the whole adult mouse brain. H\&E staining allowed for visualization of brain anatomical structures including the cerebellum (Figure 3A, i), the hippocampus (Figure 3A, ii and its inset), and the striatum (Figure 3A, iii).

We found that in the cerebellum UNC-45A is most strongly expressed in the white matter (Figure 3B, i, and its inset, red arrow), while the intensity of the staining decreases progressively in the granular (Figure 3B, i, and its inset, green arrow) and the molecular (Figure 3B, i, and its inset, red asterisks) layers of the cortex. This difference in UNC-45A staining intensity between the two cortical structures might be explained by their peculiar architecture: the granular layer is formed by neuronal bodies intercalated to the axonal fibers descending into the white matter, while the molecular layer is formed by a sparse network of dendrites, some of which stand out from the background (Figure 3B, i, next to the asterisks) due to their high UNC-45A expression. We also found that UNC-45A is abundantly expressed in the dendrites of the CA1 hippocampal neurons (Figure 3B, ii. and its inset, black arrow). In the striatum, UNC-45A is strongly expressed in the nervous bundles formed by axons crossing this grey structure (Figure 3B, iii, black arrows). To gain more details on the pattern of UNC-45A expression in the mouse brain, we performed immunofluorescence analysis on the whole mouse brain stained for UNC-45A (green), the neuronal marker NeuN [37] (magenta), and DAPI (blue) to stain nuclei (Figure 4A). As shown in Figure 4B,C, in hippocampal CA1 neurons, UNC-45A has a perinuclear and cytoplasmic localization and is particularly enriched in the dendrites (yellow arrow). Furthermore, UNC-45A was also confirmed to have strong staining in the nervous bundles of the striatum (Figure 4D, white asterisks). Quantification of UNC-45A expression levels in the brain as determined via immunofluorescence is given in Figure 4E.

Next, we performed H\&E and UNC-45A staining of the mouse spinal cord (Figure 5A upper and lower panels, respectively) and mouse nerve root (Figure 5E, upper and lower panel, respectively). In the spinal cord, UNC-45A is more abundantly expressed in the white matter (Figure 5B, i.) than in the grey matter (Figure 5B, ii). In the white matter, intense UNC-45A staining can be seen in cross-sections of single axons (Figure 5C), surrounded by UNC-45A-negative myeline sheath. Interestingly, a strong UNC-45A expression can also be found in the feet of the astrocytes forming the hematoencephalic barrier in the area 
around vessels (Figure 5B, green inset, green arrows) while the ependymocytes express UNC-45A prevalently in their cilia (Figure 5D, red asterisk), with the cytoplasm being just weakly positive. In the nerve root, the meninges (Figure 5E lower panel, black arrow) are negative for UNC-45A while a strong UNC-45A staining can be found in both, longitudinal (Figure $5 \mathrm{~F}$ ) and cross-sections (Figure $5 \mathrm{G}$ ). In the cross-section of the nerve roots, it is evident how strongly UNC-45A positive axons are surrounded by a negatively stained myelin sheath (red arrow). Quantification of UNC-45A expression levels in the mouse CNS as determined via immunohistochemistry is given in Figure 6.

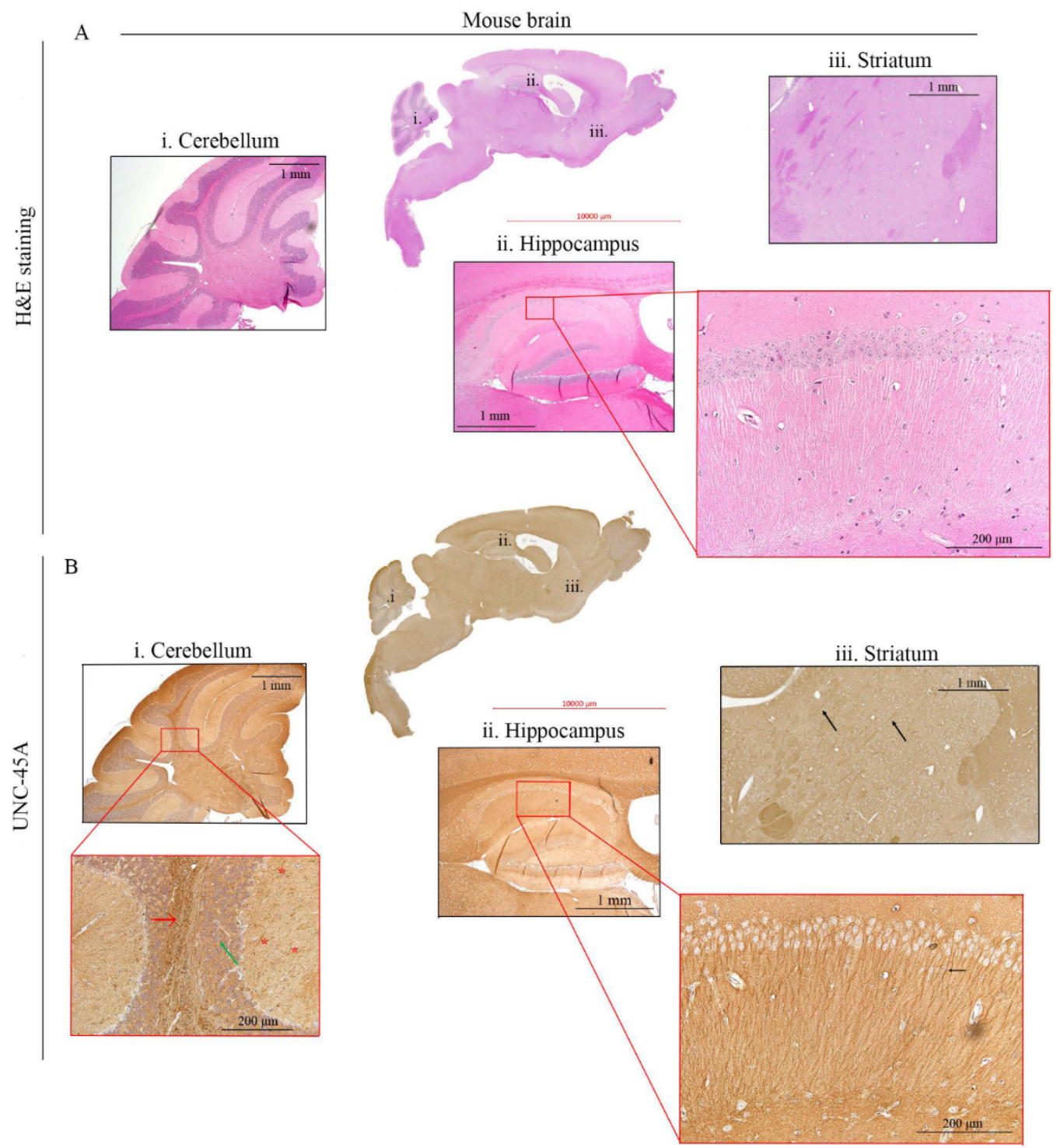

Figure 3. UNC-45A expression in mouse brain via IHC. (A). H\&E staining of the whole mouse brain. i. Cerebellum, ii. Hippocampus, iii. Striatum. (B). UNC-45A staining in the whole mouse brain. i. details on UNC-45A staining in Cerebellum (inset) from the center to the sides: white matter (red arrow) granular layer (green arrow), molecular layer (red asterisks), ii. details of UNC-45A staining in hippocampus, inset closer view on hippocampal CA1 neurons, with clearly visible dendrites (black arrow). iii. details of UNC-45A staining in the striatum, arrows indicate stronger UNC-45A staining in nervous bundles. 
Mouse brain

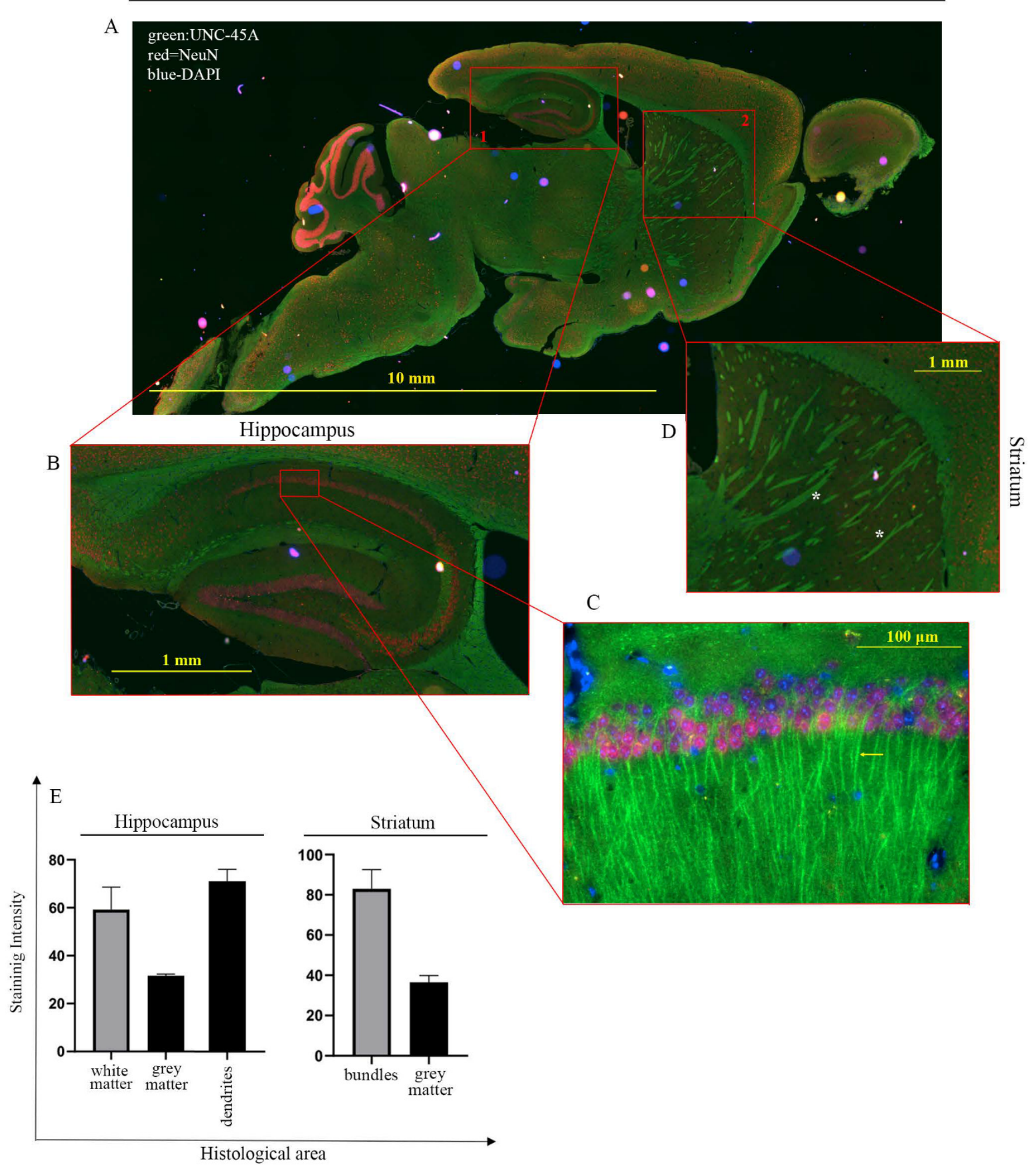

Figure 4. UNC-45A expression in mouse brain via immunofluorescence (IF). (A). Immunofluorescence staining for UNC45A (green), NeuN (neuronal nuclear protein-magenta), and DAPI (4',6-diamidino-2-phenylindole-blue) in the whole mouse brain. (B). Details of the stained hippocampus. (C). Close-up of UNC-45A staining in dendrites of hippocampal neurons (yellow arrow), DAPI staining of nuclei, and NeuN staining the neuronal soma. (D). Details of UNC-45A staining in the striatum (white asterisks indicate the nervous bundles). (E). Quantification of expression levels of UNC-45A in the indicated histological areas of the CNS. Quantification is expressed as mean pixel intensity. 


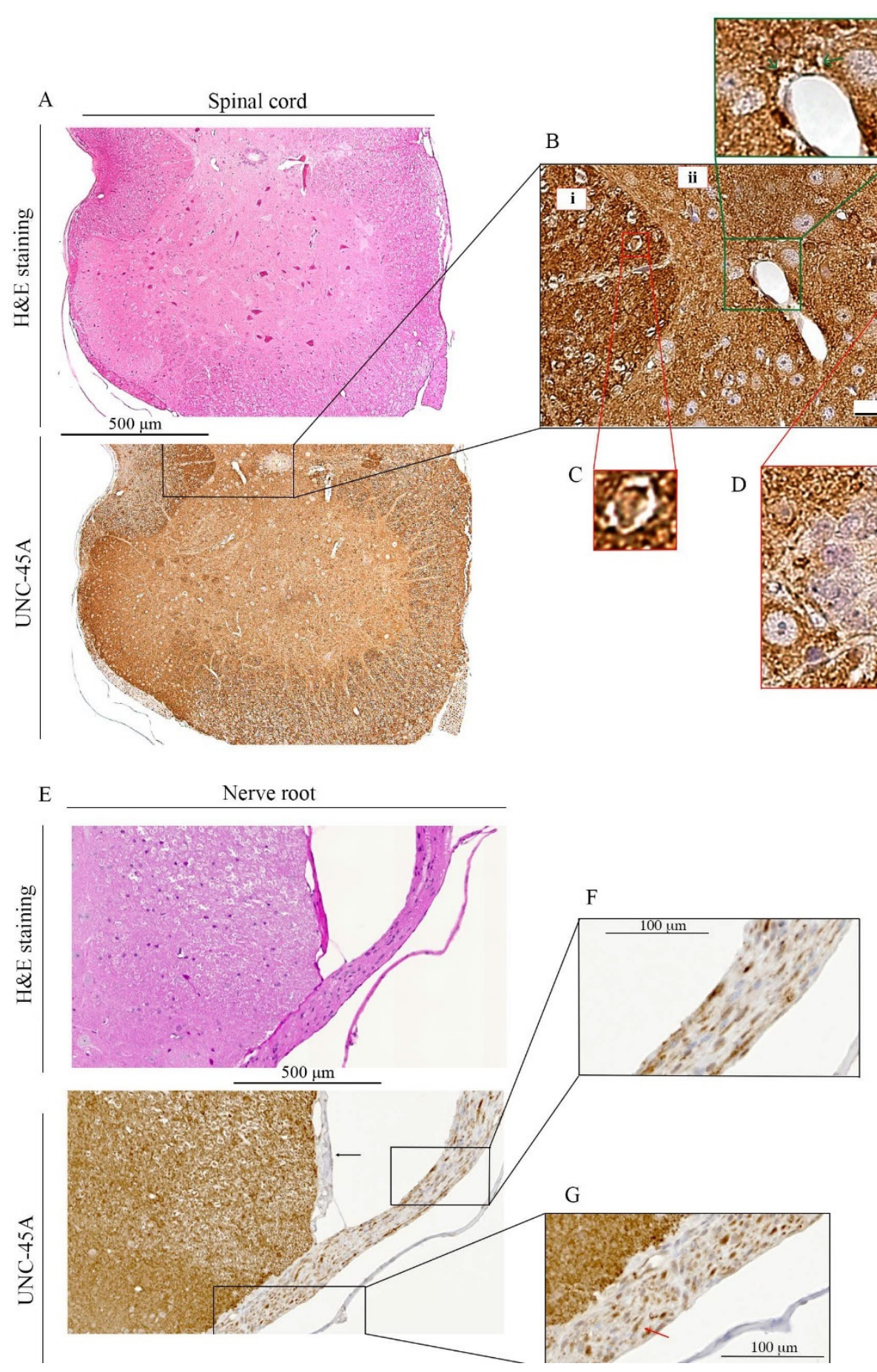

Figure 5. UNC-45A expression in mouse spinal cord and nerve root. (A). H\&E (upper panel) and UNC-45A (lower panel) staining of the mouse spinal cord. (B). Details of UNC-45A staining in white matter (i), grey matter (ii), perivascular area (green arrow). (C). Detail of UNC-45A staining of a single cross-sectioned axon with its myelin coat. (D). UNC-45A staining in ependymocytes and their ciliary structures (red asterisk). (E). H\&E (upper panel) and UNC-45A (lower panel) staining of the mouse nerve root (black arrow indicates the meninge). (F). UNC-45A staining in the longitudinal section of the nerve root. (G). UNC-45A staining in cross-section of the nerve root (red arrow indicates the myelin sheath). 


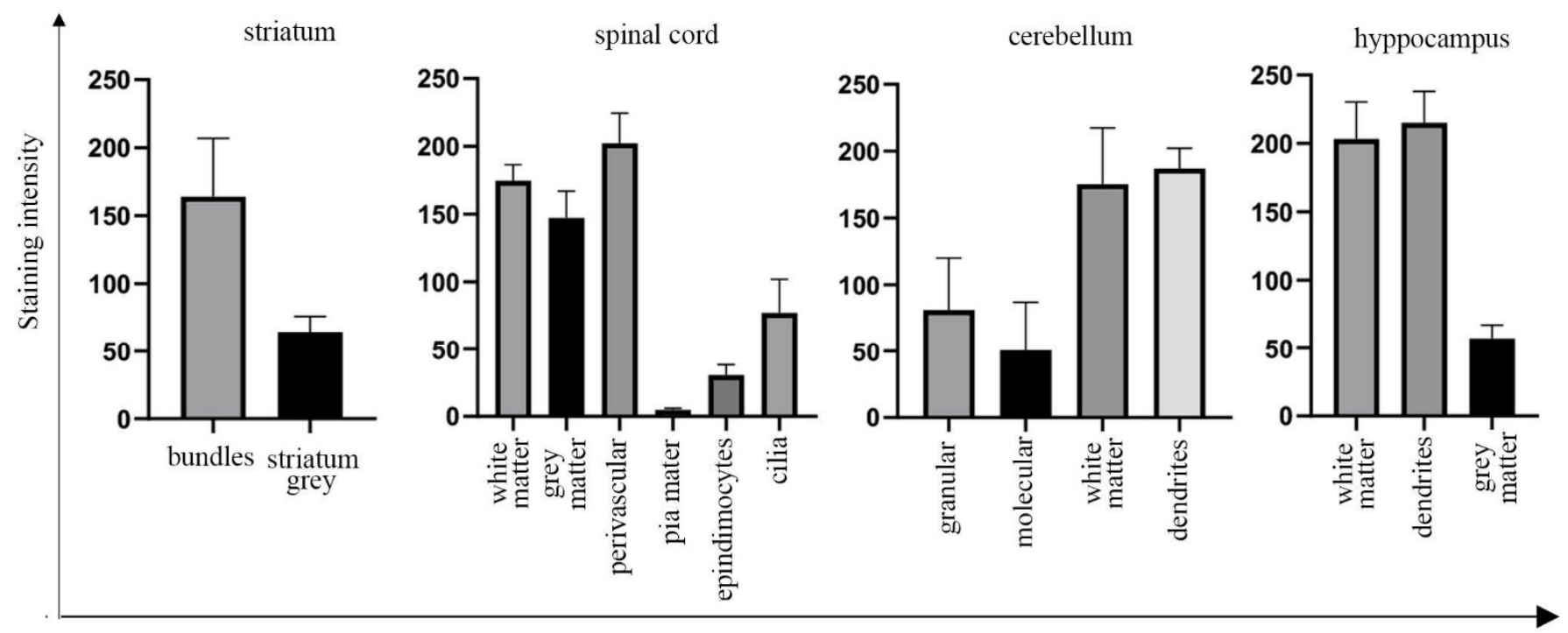

Histological area

Figure 6. Quantification of UNC-45A expression levels in the mouse central nervous system (CNS) following IHC staining. Quantification of UNC-45A staining intensity in the indicated histological areas of the CNS following IHC staining. Quantification is expressed as mean pixel intensity.

Taken together, this suggests that UNC-45A may play a relevant role in nervous system physiology. In particular, considering the crucial roles of MTs and their MAPs in neuronal biology $[35,36]$, the preferential and abundant expression of UNC-45A in axons and dendrites of mature neurons shows that this protein is not only required for their development [29], but may also be important for the neuronal functions and homeostasis. This could thus hint at a role for UNC-45A in more complex processes such as brain plasticity and/or neurodegeneration.

\section{Discussion}

Here, we found that in the mouse upper genital tract UNC-45A is enriched in proliferating and prone to remodeling cells as compared to quiescent cells. When we took into account possible variabilities in the expression of UNC-45A following the hormonal and structural changes that accompany the progression of the estrous cycle, we found no significant differences in UNC-45A expression pattern in diestrus vs. proestrus. Given that the transition from diestrus to proestrus occurs with an important hormonal shift from progesterone to estrogens, these findings suggest that UNC-45A expression could be only indirectly related to female hormones. On the other hand, considering UNC-45A known roles, minor changes may more likely be explained by the differences in proliferation that naturally occur during phase transition. Indeed, if compared to diestrus, during proestrus we found higher levels of UNC-45A in the more mature follicles, which are also more proliferative in this phase, but smaller differences were found in the corpus luteum, where UNC-45A may be still required for the cytoskeletal remodeling that occurs during cellular degeneration. Of note is that we found that UNC-45A is strongly expressed in both the oocyte and the ciliated epithelium, which plays a crucial role during fertility, as it allows for the progression of the oocyte along the fallopian tube after ovulation. These findings, along with the fact that UNC-45A is overexpressed in ovarian cancer [25] and that MT-severing proteins are involved in oocyte development [38-40], hint at the potential relevance of UNC-45A in the pathophysiology of the ovaries including reproductive biology and ovarian cancer initiation. In addition, our ex vivo observation that UNC-45A is highly expressed in proliferative cells adds strength to the importance of UNC-45A in the proliferation at the cellular level. This is consistent with previous reports [5,24-27] and, especially in ovarian cancer, UNC-45A is necessary to support the growth of cancer cells and to develop chemoresistance to paclitaxel $[5,6,25]$. The latter is a distinctive trait of 
recurrent ovarian cancer [41] and is often thought to arise from undifferentiated cancer cells (generally referred to as cancer stem-like cells) [42] and, particularly, UNC-45A expression has been inversely correlated with differentiation $[24,25,43]$.

We also found that UNC-45A is expressed in neurons in the mouse nervous system and is particularly enriched in areas containing axons and dendrites. Interestingly, Tau, a MAP that stabilizes MT via preventing MT severing [44], is largely expressed in the brain, its hyperphosphorylation is associated with Alzheimer's disease (AD) and other tauopathies [45-50], and it is abnormally expressed in ovarian cancer [51,52]. Importantly, drugs targeting MT stability are an established anti-cancer approach for ovarian cancer $[53,54]$ and stabilization of neuronal MTs can attenuate neurodegenerative in mouse models of AD and other tauopathies [55]. In this context, our results suggest that UNC-45A may play a role in the physiology of the central nervous system as well as in neurodegeneration.

Lastly, we found that UNC-45A is abundantly expressed in both the ciliated columnar epithelium of the fallopian tube and the cilia of the ependymocytes. Interestingly, congenital loss-of-function mutations in UNC-45A cause a syndrome characterized by diarrhea, cholestasis, bone fragility, and impaired hearing [56], all of which symptoms involve organs where cilia play important roles in physiology [57-59]. Furthermore, almost all of the subjects affected by congenital UNC-45A loss have signs of intellectual disability [56]. Although this may be partially due to deafness or/and other aspecific and indirect causes associated with the syndrome, it is also possible that UNC-45A loss may be directly responsible for the intellectual disability of these patients given its wide expression in the central nervous system and its requirement for the extension of neurites [29]. Taken together, our results also suggest that UNC-45A may play a role in the physiology of ciliated epithelia as well as in ciliopathies.

Author Contributions: Conceptualization, M.B., V.C. and A.H.; methodology, V.C., A.H. and J.M.; software, V.C., A.H. and J.M.; validation, V.C., A.H. and M.B.; formal analysis, V.C., A.H., M.B.; investigation, V.C., A.H. and M.B.; resources, T.S., M.L., M.S., J.M. and M.B.; data curation, V.C., A.H. and M.B.; writing—original draft preparation, V.C., A.H., M.B., M.S. and J.M.; writing-review and editing, V.C., A.H., M.B. and M.S.; visualization, V.C., A.H., M.B. and J.M.; supervision, M.B., M.L., T.S.; project administration, M.B.; funding acquisition, M.B., T.S. All authors have read and agreed to the published version of the manuscript.

Funding: This work was supported by the US Department of Defense Ovarian Cancer Research Program (OC160377), the Minnesota Ovarian Cancer Alliance, the Randy Shaver Cancer Research and Community Fund and the National Institute of General Medical Sciences (R01-GM130800) to M.B. This work was supported by the Minnesota Ovarian Cancer Alliance and the Masonic Cancer Center to T.S. This work was supported by Rotary Club Forlì to V.C. The funders had no role in study design, data collection and analysis, decision to publish or preparation of the manuscript.

Institutional Review Board Statement: The study was conducted according to the guidelines of the Declaration of Helsinki, and approved by the University of Minnesota's Institutional Animal Care and Use Committee (protocol N2003-37929A, approved on 27 March 2020, for the female genital tract specimens; protocol \#1902-36759A, approved on 12 March 2019 and 18 February 2021, for the CNS specimens).

Informed Consent Statement: Not applicable.

Conflicts of Interest: The authors declare no conflict of interest.

\section{References}

1. Barral, J.M.; Hutagalung, A.H.; Brinker, A.; Hartl, F.U.; Epstein, H.F. Role of the myosin assembly protein UNC-45 as a molecular chaperone for myosin. Science 2002, 295, 669-671. [CrossRef]

2. Kachur, T.; Ao, W.; Berger, J.; Pilgrim, D. Maternal UNC-45 is involved in cytokinesis and colocalizes with non-muscle myosin in the early Caenorhabditis elegans embryo. J. Cell Sci. 2004, 117, 5313-5321. [CrossRef]

3. Shi, H.; Blobel, G. UNC-45/CRO1/She4p (UCS) protein forms elongated dimer and joins two myosin heads near their actin binding region. Proc. Natl. Acad. Sci. USA 2010, 107, 21382-21387. [CrossRef] 
4. Lehtimäki, J.; Fenix, A.; Kotila, T.M.; Balistreri, G.; Paavolainen, L.; Varjosalo, M.; Burnette, D.T.; Lappalainen, P. UNC-45a promotes myosin folding and stress fiber assembly. J. Cell Biol. 2017, 216, 4053-4072. [CrossRef] [PubMed]

5. Habicht, J.; Mooneyham, A.; Shetty, M.; Zhang, X.; Shridhar, V.; Winterhoff, B.; Zhang, Y.; Cepela, J.; Starr, T.; Lou, E.; et al. UNC-45A is preferentially expressed in epithelial cells and binds to and co-localizes with interphase MTs. Cancer Biol. Ther. 2019, 20, 1304-1313. [CrossRef] [PubMed]

6. Mooneyham, A.; Iizuka, Y.; Yang, Q.; Coombes, C.; McClellan, M.; Shridhar, V.; Emmings, E.; Shetty, M.; Chen, L.; Ai, T.; et al. UNC-45A is a novel microtubule-associated protein and regulator of paclitaxel sensitivity in ovarian cancer cells. Mol. Cancer Res. 2019, 17, 370-383. [CrossRef]

7. Habicht, J.; Mooneyham, A.; Hoshino, A.; Shetty, M.; Zhang, X.; Emmings, E.; Yang, Q.; Coombes, C.; Gardner, M.K.; Bazzaro, M. UNC-45A breaks mt lattice independent of its effect on non-muscle myosin II. J. Cell Sci. 2021. [CrossRef]

8. Sarwar, M.; Sykes, P.H.; Chitcholtan, K.; Evans, J.J. Deciphering biophysical modulation in ovarian cancer cells. Cell Biophys. 2021, 79, 375-386. [CrossRef]

9. McGrail, D.J.; Kieu, Q.M.N.; Dawson, M.R. Metastatic ovarian cancer cell malignancy is increased on soft matrices through a mechanosensitive Rho/ROCK pathway. J. Cell Sci. 2014, 127, 2621-2626. [CrossRef]

10. Jeong, K.J.; Park, S.Y.; Cho, K.H.; Sohn, J.S.; Lee, J.; Kim, Y.K.; Kang, J.; Park, C.G.; Han, J.W.; Lee, H.Y. The Rho/ROCK pathway for lysophosphatidic acid-induced proteolytic enzyme expression and ovarian cancer cell invasion. Oncogene 2012, 31, 4279-4289. [CrossRef]

11. Ohta, T.; Takahashi, T.; Shibuya, T.; Amita, M.; Henmi, N.; Takahashi, K.; Kurachi, H. Inhibition of the Rho/ROCK pathway enhances the efficacy of cisplatin through the blockage of hypoxia-inducible factor- $1 \alpha$ in human ovarian cancer cells. Cancer Biol. Ther. 2012, 13, 25-33. [CrossRef] [PubMed]

12. Mehra, A.; Guérit, S.; Macrez, R.; Gosselet, F.; Sevin, E.; Lebas, H.; Maubert, E.; De Vries, H.E.; Bardou, I.; Vivien, D.; et al. Nonionotropic Action of Endothelial NMDA Receptors on Blood-Brain Barrier Permeability via Rho/ROCK-Mediated Phosphorylation of Myosin. J. Neurosci. 2020, 40, 1778-1787. [CrossRef]

13. Stankiewicz, T.R.; Pena, C.; Bouchard, R.J.; Linseman, D.A. Dysregulation of Rac or Rho elicits death of motor neurons and activation of these GTPases is altered in the G93A mutant hSOD1 mouse model of amyotrophic lateral sclerosis. Neurobiol. Dis. 2020, 136, 104743. [CrossRef]

14. Denton, K.R.; Lei, L.; Grenier, J.; Rodionov, V.; Blackstone, C.; Li, X.-J. Loss of spastin function results in disease-specific axonal defects in human pluripotent stem cell-based models of hereditary spastic paraplegia. Stem Cells 2014, 32, 414-423. [CrossRef]

15. Tao, J.; Feng, C.; Rolls, M.M. The microtubule severing protein fidgetin acts after dendrite injury to promote degeneration. J. Cell Sci. 2016, 129, 3274-3281. [CrossRef] [PubMed]

16. Fassier, C.; Fréal, A.; Gasmi, L.; Delphin, C.; Martin, D.T.; De Gois, S.; Tambalo, M.; Bosc, C.; Mailly, P.; Revenu, C.; et al. Motor axon navigation relies on Fidgetin-like 1-driven microtubule plus end dynamics. J. Cell Biol. 2018, 217, 1719-1738. [CrossRef] [PubMed]

17. Ahmad, F.; Yu, W.; McNally, F.J.; Baas, P.W. An essential role for katanin in severing microtubules in the neuron. J. Cell Biol. 1999, 145, 305-315. [CrossRef] [PubMed]

18. Karabay, A.; Yu, W.; Solowska, J.M.; Baird, D.H.; Baas, P.W. Axonal growth is sensitive to the levels of katanin, a protein that severs microtubules. J. Neurosci. 2004, 24, 5778-5788. [CrossRef]

19. Schiewek, J.; Schumacher, U.; Lange, T.; Joosse, S.A.; Wikman, H.; Pantel, K.; Mikhaylova, M.; Kneussel, M.; Linder, S.; Schmalfeldt, B.; et al. Clinical relevance of cytoskeleton associated proteins for ovarian cancer. J. Cancer Res. Clin. Oncol. 2018, 144, 2195-2205. [CrossRef]

20. Price, D.K.; Ball, J.R.; Bahrani-Mostafavi, Z.; Vachris, J.C.; Kaufman, J.; Naumann, R.W.; Higgins, R.V.; Hall, J.B. The phosphoprotein Op18/stathmin is differentially expressed in ovarian cancer. Cancer Investig. 2000, 18, 722-730. [CrossRef]

21. Su, D.; Smith, S.; Preti, M.; Schwartz, P.; Rutherford, T.J.; Menato, G.; Danese, S.; Ma, S.; Yu, H.; Katsaros, D. Stathmin and tubulin expression and survival of ovarian cancer patients receiving platinum treatment with and without paclitaxel. Cancer 2009, 115, 2453-2463. [CrossRef] [PubMed]

22. Lombino, F.L.; Muhia, M.; Lopez-Rojas, J.; Brill, M.S.; Thies, E.; Ruschkies, L.; Lutz, D.; Richter, M.; Hausrat, T.J.; Lopes, A.; et al. The microtubule severing protein katanin regulates proliferation of neuronal progenitors in embryonic and adult neurogenesis. Sci. Rep. 2019, 9, 15940. [CrossRef] [PubMed]

23. Balachandran, R.; Welsh, M.J.; Day, B.W. Altered levels and regulation of stathmin in paclitaxel-resistant ovarian cancer cells Oncogene 2003, 22, 8924-8930. [CrossRef] [PubMed]

24. Guo, W.; Chen, D.; Fan, Z.; Epstein, H.F. Differential turnover of myosin chaperone UNC-45a isoforms increases in metastatic human breast cancer. J. Mol. Biol. 2011, 412, 365-378. [CrossRef] [PubMed]

25. Bazzaro, M.; Santillan, A.; Lin, Z.; Tang, T.; Lee, M.K.; Bristow, R.E.; Shih, I.-M.; Roden, R.B. Myosin II co-chaperone general cell UNC-45 overexpression is associated with ovarian cancer, rapid proliferation, and motility. Am. J. Pathol. 2007, 171, 1640-1649. [CrossRef]

26. Eisa, N.H.; Jilani, Y.; Kainth, K.; Redd, P.; Lu, S.; Bougrine, O.; Sater, H.A.; Patwardhan, C.A.; Shull, A.; Shi, H.; et al. The co-chaperone UNC45A is essential for the expression of mitotic kinase NEK7 and tumorigenesis. J. Biol. Chem. 2019, 294, 5246-5260. [CrossRef] 
27. Jilani, Y.; Lu, S.; Lei, H.; Karnitz, L.M.; Chadli, A. UNC45A localizes to centrosomes and regulates cancer cell proliferation through ChK1 activation. Cancer Lett. 2015, 357, 114-120. [CrossRef]

28. Iizuka, Y.; Cichocki, F.; Sieben, A.J.; Sforza, F.; Karim, R.; Coughlin, K.; Vogel, R.; Gavioli, R.; McCullar, V.; Lenvik, T.; et al. UNC-45A Is a nonmuscle myosin IIA chaperone required for NK cell cytotoxicity via control of lytic granule secretion. J. Immunol. 2015, 195, 4760-4770. [CrossRef]

29. Iizuka, Y.; Mooneyham, A.; Sieben, A.; Chen, K.; Maile, M.; Hellweg, R.; Schütz, F.; Teckle, K.; Starr, T.; Thayanithy, V.; et al. UNC-45A is required for neurite extension via controlling NMII activation. Mol. Biol. Cell 2017, 28, 1337-1346. [CrossRef]

30. Ajayi, A.F.; Akhigbe, R.E. Staging of the estrous cycle and induction of estrus in experimental rodents: An update. Fertil. Res. Pr. 2020, 6, 1-15. [CrossRef]

31. Westwood, F.R. The female rat reproductive cycle: A practical histological guide to staging. Toxicol. Pathol. 2008, 36, 375-384. [CrossRef]

32. Crowe, A.R.; Yue, W. Semi-quantitative determination of protein expression using immunohistochemistry staining and analysis: An integrated protocol. Bio-protocol 2019, 9, e3465. [CrossRef] [PubMed]

33. Marshall, W.F. The cell biological basis of ciliary disease. J. Cell Biol. 2008, 180, 17-21. [CrossRef] [PubMed]

34. Ishikawa, T.; Ueno, H.; Omori, T.; Kikuchi, K. Cilia and centrosomes: Ultrastructural and mechanical perspectives. Semin. Cell Dev. Biol. 2021, 110, 61-69. [CrossRef]

35. Kapitein, L.C.; Hoogenraad, C.C. Building the neuronal microtubule cytoskeleton. Neuron 2015, 87, 492-506. [CrossRef]

36. Dehmelt, L.; Halpain, S. The MAP2/Tau family of microtubule-associated proteins. Genome Biol. 2004, 6, 204. [CrossRef]

37. Gusel'nikova, V.V.; Korzhevskiy, D.E. NeuN as a neuronal nuclear antigen and neuron differentiation marker. Acta Naturae. 2015, 7, 42-47. [CrossRef] [PubMed]

38. Gao, L.; Xu, F.; Jin, Z.; Ying, X.; Liu, J. Microtubule-severing protein Katanin p60 ATPase-containing subunit A-like 1 is involved in pole-based spindle organization during mouse oocyte meiosis. Mol. Med. Rep. 2019, 20, 3573-3582. [CrossRef]

39. Yang, H.-Y.; McNally, K.; McNally, F.J. MEI-1/katanin is required for translocation of the meiosis I spindle to the oocyte cortex in C. elegans. Dev. Biol. 2003, 260, 245-259. [CrossRef]

40. McNally, K.; Audhya, A.; Oegema, K.; McNally, F.J. Katanin controls mitotic and meiotic spindle length. J. Cell Biol. 2006, 175, 881-891. [CrossRef] [PubMed]

41. Garzon, S.; Laganà, A.S.; Casarin, J.; Raffaelli, R.; Cromi, A.; Franchi, M.; Barra, F.; Alkatout, I.; Ferrero, S.; Ghezzi, F. Secondary and tertiary ovarian cancer recurrence: What is the best management? Gland. Surg. 2020, 9, 1118-1129. [CrossRef]

42. Lagana, A.S.; Colonese, F.; Colonese, E.; Sofo, V.; Salmeri, F.M.; Granese, R.; Chiofalo, B.; Ciancimino, L.; Triolo, O. Cytogenetic analysis of epithelial ovarian cancer's stem cells: An overview on new diagnostic and therapeutic perspectives. Eur. J. Gynaecol. Oncol. 2015, 36, 495-505.

43. Price, M.G.; Landsverk, M.L.; Barral, J.M.; Epstein, H.F. Two mammalian UNC-45 isoforms are related to distinct cytoskeletal and muscle-specific functions. J. Cell Sci. 2002, 115, 4013-4023. [CrossRef] [PubMed]

44. Tan, R.; Lam, A.J.; Tan, T.; Han, J.; Nowakowski, D.W.; Vershinin, M.; Simó, S.; Ori-McKenney, K.M.; McKenney, R.J. Microtubules gate tau condensation to spatially regulate microtubule functions. Nat. Cell Biol. 2019, 21, 1078-1085. [CrossRef] [PubMed]

45. Duan, Y.; Dong, S.; Gu, F.; Hu, Y.; Zhao, Z. Advances in the pathogenesis of Alzheimer's Disease: Focusing on tau-mediated neurodegeneration. Transl. Neurodegener. 2012, 1, 24. [CrossRef] [PubMed]

46. Baas, P.W.; Rao, A.; Matamoros, A.J.; Leo, L. Stability properties of neuronal microtubules. Cytoskelet. 2016, 73, 442-460. [CrossRef] [PubMed]

47. Dubey, J.; Ratnakaran, N.; Koushika, S.P. Neurodegeneration and microtubule dynamics: Death by a thousand cuts. Front. Cell. Neurosci. 2015, 9, 343. [CrossRef]

48. Hellal, F.; Hurtado, A.; Ruschel, J.; Flynn, K.C.; Laskowski, C.J.; Umlauf, M.; Kapitein, L.C.; Strikis, D.; Lemmon, V.; Bixby, J.; et al. Microtubule stabilization reduces scarring and causes axon regeneration after spinal cord injury. Science 2011, 331, $928-931$. [CrossRef]

49. Jean, D.C.; Baas, P.W. It cuts two ways: Microtubule loss during Alzheimer disease. EMBO J. 2013, 32, 2900-2902. [CrossRef]

50. Ruschel, J.; Hellal, F.; Flynn, K.C.; Dupraz, S.; Elliott, D.A.; Tedeschi, A.; Bates, M.; Sliwinski, C.; Brook, G.; Dobrindt, K.; et al. Systemic administration of epothilone B promotes axon regeneration after spinal cord injury. Science 2015, 348, 347-352. [CrossRef]

51. Mimori, K.; Sadanaga, N.; Yoshikawa, Y.; Ishikawa, K.; Hashimoto, M.; Tanaka, F.; Sasaki, A.T.; Inoue, H.; Sugimachi, K.; Mori, M. Reduced tau expression in gastric cancer can identify candidates for successful Paclitaxel treatment. Br. J. Cancer 2006, 94, 1894-1897. [CrossRef]

52. Plun-Favreau, H.; Lewis, P.A.; Hardy, J.; Martins, L.M.; Wood, N.W. Cancer and Neurodegeneration: Between the Devil and the Deep Blue Sea. PLoS Genet. 2010, 6, e1001257. [CrossRef]

53. Owinsky, E.R.K.R.; Onehower, R.O.C.D. Paclitaxel (Taxol). N. Engl. J. Med. 1995, 332, 1004-1014. [CrossRef] [PubMed]

54. Bernabeu, E.; Cagel, M.; Lagomarsino, E.; Moretton, M.; Chiappetta, D.A. Paclitaxel: What has been done and the challenges remain ahead. Int. J. Pharm. 2017, 526, 474-495. [CrossRef]

55. Duggal, P.; Mehan, S. Neuroprotective approach of anti-cancer microtubule stabilizers against tauopathy associated dementia: Current status of clinical and preclinical findings. J. Alzheimer's Dis. Rep. 2019, 3, 179-218. [CrossRef] 
56. Esteve, C.; Francescatto, L.; Tan, P.L.; Bourchany, A.; De Leusse, C.; Marinier, E.; Blanchard, A.; Bourgeois, P.; Brochier-Armanet, C.; Bruel, A.-L.; et al. Loss-of-function mutations in UNC45A cause a syndrome associating cholestasis, diarrhea, impaired hearing, and bone fragility. Am. J. Hum. Genet. 2018, 102, 364-374. [CrossRef]

57. Lee, L.; Ostrowski, L.E. Motile cilia genetics and cell biology: Big results from little mice. Cell. Mol. Life Sci. 2021, 78, $769-797$. [CrossRef]

58. Suciu, S.K.; Caspary, T. Cilia, neural development and disease. Semin. Cell Dev. Biol. 2021, 110, 34-42. [CrossRef]

59. Higgins, M.; Obaidi, I.; McMorrow, T. Primary cilia and their role in cancer (Review). Oncol. Lett. 2019, 17, 3041-3047. [CrossRef] 